\title{
Das Liebig-Museum in Gießen
}

Das Liebig-Museum ist erst zu Beginn dieses Jahrhunderts geschaffen worden. Durch die großzügige Unterstützung des Großherzogs Ludwig I. (siehe Auslage im Museum) war Liebig der heißersehnte Wunsch, in Paris sein Studium fortzusetzen, erfüllt worden. Alexander von Humboldt brachte den jungen Studenten in Verbindung mit den Gelehrten der Naturwissenschaften an der Sorbonne. Schließlich empfahl Humboldt den 21jährigen Chemiker dessen Landesherrn mit Schreiben vom 5. Februar 1824 (in deutscher Utbersetzung): "Wir haben den Vorzug gehabt, unter uns einen Ihrer Untertanen zu sehen, Monseigneur, der durch die Uberlegenheit seines Talents, der ausgedehnten Kenntnisse in der Chemie und seinen Scharfsinn die lebhafte Aufmerksamkeit des Instituts Royal de France auf sich gezogen hat; der Dr. Liebig verbindet mit den Gaben des Geistes eine Liebenswürdigkeit des Charakters und Feinheit der Sitten, wie sie unter Gelehrten seiner Zeit so selten ist. Wenn meine schwache Stimme von einigem Gewicht sein könnte, würde ich Eure Königliche Hoheit ergebenst bitten, Monsieur Liebig auch fernerhin die Gunst Ihrer besonderen Protektion angedeihen zu lassen. Er wird ein Professor sein, der unserem Vaterland Ehre macht, und die lebhafte Erkenntlichkeit, von der ich durchdrungen bin für einen Souverän, der die Güte hat, ein so ausgezeichnetes Talent zu ehren, wird geteilt von meinen Kollegen an der Akademie, den Herren Gay-Lussac, Thénard, Dulong und Vauquelin... “

Mit Decret vom 26. Mai 1824 wurde Liebig zum außerordentlichen Professor an der Hessischen Landesuniversität ernannt. Als Raum für Laboratorien und zum Vortrag wurde ihm das Wachlokal einer ehemaligen Kaserne auf dem Seltersberg zugewiesen. Bereits am 7. November begann Liebig seine ersten Vorlesungen mit 12 Schülern. Im Jahre 1832 wurde ein Anbau errichtet, in welchem Liebig ein eigenes Studierzimmer und ein Privatlaboratorium erhielt. 1839 erfuhr das Institut eine beachtliche Erweiterung (Querbau) durch zwei geräumige Laboratorien, eine Bibliothek und einen Hörsaal für etwa 80 Studierende. Im Dachgeschoß hatte Liebig seine Privatwohnung.

Die Aufteilung der Räume (nach vorhandenen Plänen des Architekten J. Ph. Hofmann, dem Vater von Liebigs Schüler A. W. Hofmann) war nach Fertigstellung des letzten Anbaues folgende: Hinter dem Säulenportal befand sich das sogenannte "Alte Laboratorium"; daran schlossen sich an, rechts, das „Zimmer für den Diener im Laboratorium" und das "Waarenzimmer", links die "Waschküche" und das „Zimmer zum Aufbewahren der Praeparate“. Es folgten das „Laboratorium und ein Kabinett für den Direktor", weiter das „Pharmaceutische Laboratorium“, "Instrumenten- und Bibliothekzimmer" und "Waagenzimmer" und schlieBlich im Querbau das "Analytische Laboratorium" (Wiedergabe desselben im Deutschen Museum) und das „Auditorium“. Dieses Laboratorium wurde zum Vorbild oder, wie Liebigs Biograph Jacob Volhard schreibt, zur Mutter aller chemischen Institute. So bestand es bis zum Jahre 1888, also noch 36 Jahre nach Liebigs Uber- 
siedlung nach München. Damals wurde in Gießen ein den modernen Ansprüchen entsprechendes Institut erbaut.

Der größere Teil des ehemaligen Liebig'schen Laboratoriums diente nun als Lehrstätte für Bakteriologie und Hygiene, während das ehemalige Auditorium für Küchenzwecke der benachbarten Universitätsklinik Verwendung fand. Später zog das Untersuchungsamt Oberhessen in das alte Labor ein, und schließlich wurden chemisch-physikalische Vorlesungen mit Praktikum dort abgehalten. Im Jahre 1898 drohte sogar infolge neuer Bahnanlagen die Gefahr des Abbruchs des Gebäudes. Später erwog man den Plan, ein Zahnärztliches Institut darin einzurichten. Bereits im Jahre 1909 hatte der Geheime Medizinalrat Professor Dr. Robert Sommer sich an die zuständigen Behörden mit dem Vorschlag gewandt, die Räume des früheren Liebig-Laboratoriums zur Einrichtung eines Liebig-Museums freizugeben. Er fand damals kein Verständnis.

Zu Beginn des Jahres 1910 schlossen sich indessen begeisterte Anhänger seiner Idee zu einem „Arbeitsausschuß zur Erhaltung des Gießener Liebig-Laboratoriums“ zusammen; noch im selben Jahr wurde die „Gesellschaft Liebig-Museum“ gegründet. Der damalige Großherzog Ernst Ludwig hatte das Protektorat übernommen. Der erste Aufruf erweckte allgemeines Interesse und brachte auch wirtschaftlichen Erfolg.

Manche Schwierigkeit war zu überwinden. Es ist zweifellos das große Verdienst von Geheimrat Sommer, der unermüdlich, - erstmalig, wie bereits erwähnt, im Jahre 1909, - öffentlich auftrat, durch Reden und Aufsätze in Zeitschriften sich zähe und verbissen für die Erhaltung des Liebig-Laboratoriums und dessen spätere Wiedereinrichtung als Museum eingesetzt zu haben. Mit ihm zusammen arbeitete mit gleicher Energie und Tatkraft der Geheime Medizinalrat Dr. E. A. Merck. Als die Stadt entgegenkommenderweise für den Erwerb von Grundstück und Gebäude, jedoch mit einem Zahlungstermin von 24 Tagen, den Betrag von nur 60 000.- Mark forderte, der keineswegs zur Verfügung stand, garantierte er denselben und schenkte ihn später der Gesellschaft.

Nach Instandsetzung des Gebäudes galt es nun, die Räume so wieder einzurichten, wie es zu Liebigs Zeiten gewesen war. Aber nur wenige alte Einrichtungsstücke waren noch vorhanden. Das meiste mußte rekonstruiert werden. Schrittweise ging man vor. Alte Pläne des Architekten J. Ph. Hofmann dienten als Unterlage. So fand man $u$. a. den in der Zwischenzeit zugemauerten Kaliofen im analytischen Laboratorium wieder. Ein wertvoller Zuwachs war eine Reihe von Originaleinrichtungen aus Liebigs Zeiten, andere Teile mußten als Nachbildung ergänzt werden. Die Gesellschaft erhielt von Angehörigen der Familie Liebig, von Freunden und ehemaligen Schülern Geschenke aller Art, Schriften, Bücher, Briefe und sonstige Gegenstände; so u. a. den Schreibtisch aus der Gießener Zeit sowie das Zeitungsgestell.

Frau Geheimrat A. W. v. Hofmann stiftete 90 außerordentlich wichtige Briefe von: Liebig an ihren verstorbenen Gatten. 
Die Laboratorien, das Auditorium sowie Liebigs Privatlabor und Arbeitszimmer konnten im wesentlichen so wieder eingerichtet werden, teils mit Originalstücken, teils ergänzt, wie sie zur Zeit des Meisters gewesen waren.

Am 26. März 1920 wurde das Museum feierlich eröffnet und zieht seitdem einen ständig steigenden Strom von Besuchern aus aller Welt, Wissenschaftlern wie Laien, in seine Mauern. Auch die Zahl der Mitglieder der Gesellschaft Liebig. Museum ist seit dieser Zeit in erfreulichem Steigen begriffen. Unterbrochen wurde diese günstige Entwicklung nur im zweiten Weltkrieg und den Jahren danach, als das Museum, das gegen Ende des Krieges durch Fliegereinwirkung besonders im vorderen Teil des Gebäudes stark gelitten hatte, für einige Zeit seine Pforten schließen mußte. In großzügiger Weise ist durch den Magistrat der Stadt Gießen der größte Schaden sofort behoben worden. Mit weiterer Unterstützung des Magistrats sowie durch einen Aufruf an die chemische Industrie der Bundesrepublik und Schweizer chemische Fabriken, konnte später das Gebäude wieder vollständig instandgesetzt werden.

Am 1. Juli 1952 wurde das Museum wieder eröffnet. Zu dieser Feier waren 15 Angehörige der Familie Liebig erschienen, darunter Dr. Heinrich Freiherr v. Liebig, heute der letzte Träger des Freiherrlichen Namens. Im Jahre darauf wurde gemeinsam mit der Stadt Gießen und der Justus Liebig-Hochschule der 150. Geburtstag des Meisters gefeiert.

Glücklicherweise erhielt das Museum auch in den letzten Jahren noch wesentliche Bereicherungen. Der inzwischen verstorbene Justizrat Dr. Justus Freiherr v. Liebig schenkte der Gesellschaft die Marmorbüste seines Großvaters. Dr. Heinrich Freiherr v. Liebig überließ uns den Schreibtisch seines Großvaters aus der Münchener Zeit, dazu eine Sammlung wertvoller Bücher, die Liebig persönlich benutzte, u. a. den Gmelin. Von Herrn Carl Liebig aus Valparaiso, einem Großneffen Liebigs, erhielten wir eine große Anzahl interessanter Briefe sowie das 'Tagebuch der "Tiroler Reise". Der Herr Bundespräsident hat sein besonderes Interesse für das Museum durch Stiftung einer Reihe von Briefen Liebigs an seine Schwester, die Großmutter der verstorbenen Frau Elly Heuß-Knapp, bekundet. Ferner erhielten wir von vielen weiteren Nachkommen Liebigs Briefe, Photographien und manche andere schöne Erinnerungsstücke.

Zur Wiedereinrichtung des zerstörten „Alten Labors“ überließ uns das Deutsche Apotheken-Museum in Bamberg eine Anzahl alte Retorten, Destillierapparate und Schmelztiegel, wie sie zur Zeit Liebigs verwendet wurden. Bei Auflösung der „Vereinigung Liebig-Haus e. V." Darmstadt im Juli 1950 übernahm die Gießener Gesellschaft deren Bestand an alten Akten und Briefen.

Die Bibliothek des Museums enthält sämtliche Werke des Meisters, seine Schriften und Reden, die seine rastlose Arbeitskraft und seinen unermüdlichen Fleiß bezeugen, - überstrahlt aber von einem genialen Geist. Die Briefe und Dokumente sind von Fräulein Dr. Hertha von Dechend (Verfasserin von „Justus von Liebig in eigenen Zeugnissen und solchen seiner Zeitgenossen". Verlag Chemie 1953.), Mit- 
arbeiterin des Instituts für Geschichte der Naturwissenschaften der Johann-Wolfgang-Goethe-Universität (Direktor: Professor Dr. Willy Hartner) neu geordnet und registriert worden. Ferner hat Fräulein von Dechend von dem wichtigsten Teil des in der Bayerischen Staatsbibliothek aufbewahrten Liebig-Nachlasses Mikrofilme angefertigt. Ob wir noch weiterhin Briefe von und an Liebig sowie den Gelehrten betreffende Gegenstände erwerben können, überlassen wir der Zukunft. Der derzeitige Vorstand ist sich, getreu seinen Vorgängern, als Hüter des Museums seiner verantwortungsvollen Aufgabe bewuBt:

DEM ANDENKEN LIEBIGS. 


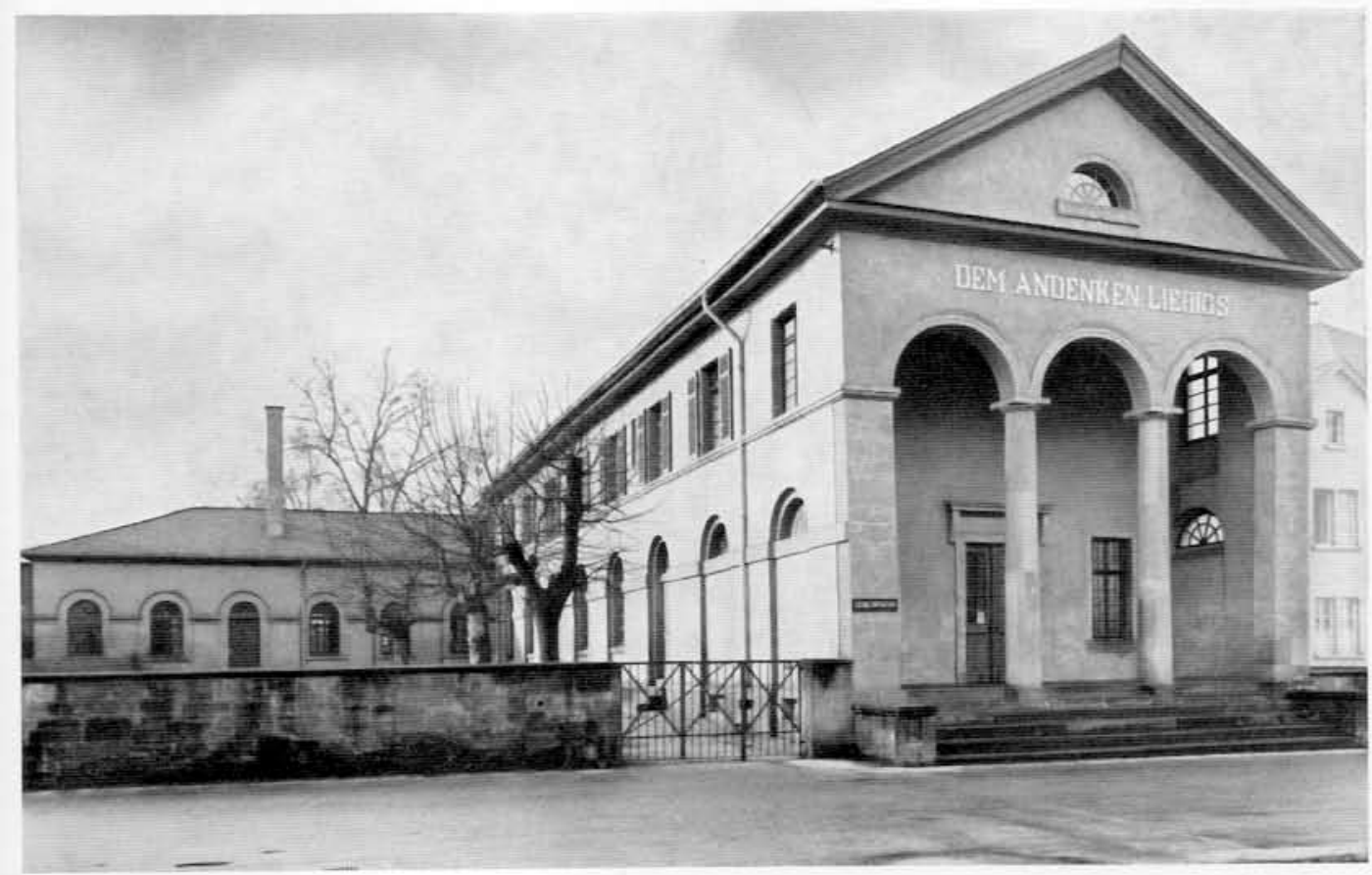

Liebigs Arbeitsstätte in Gießen Jetzt Liebig-Museum 


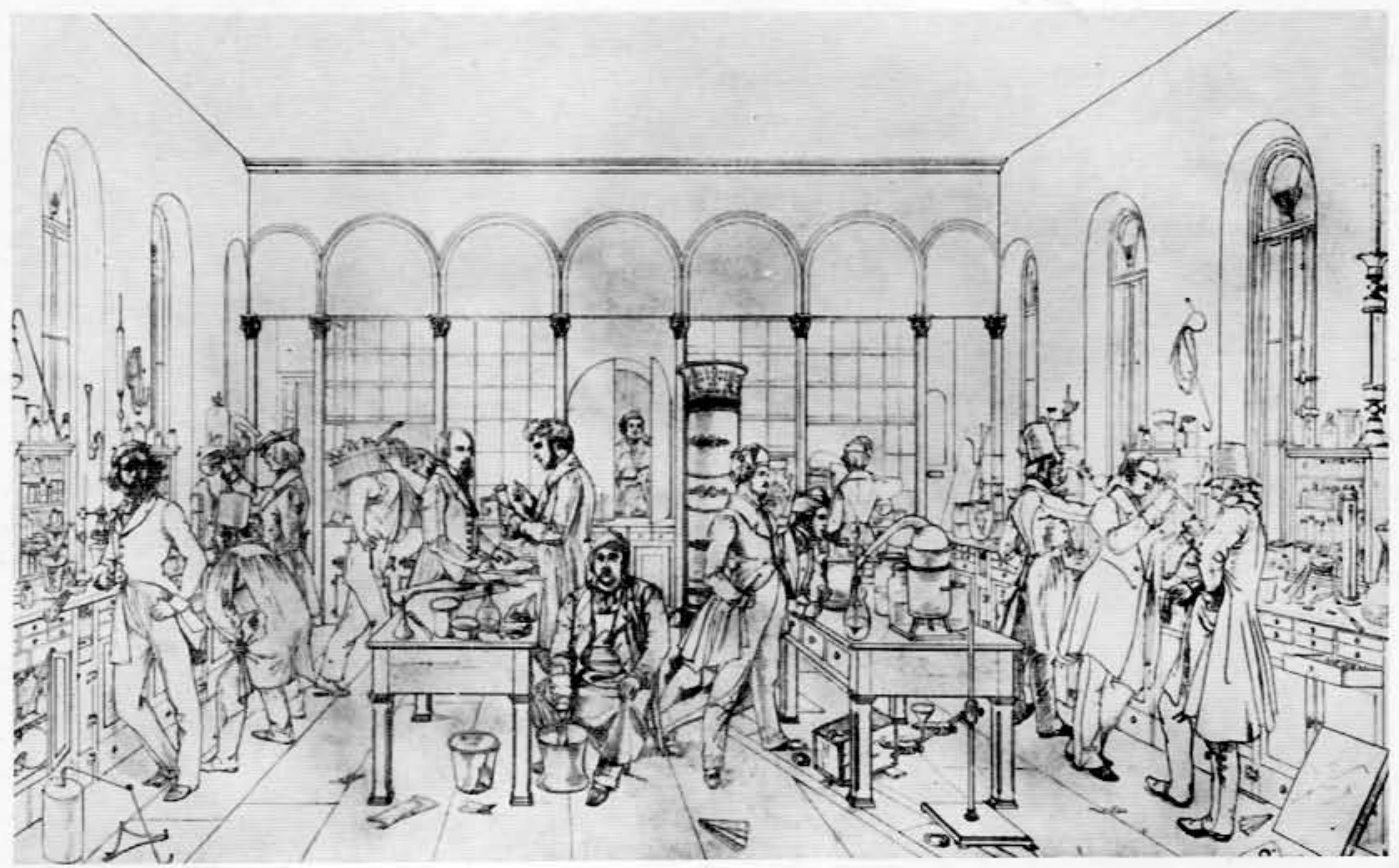

Justus Liebigs Laboratorium in Gießen 